\title{
Posicionador radiográfico periapical para pacientes com discrepâncias intermaxilares severas
}

\section{Periapical Radiographic Positioner for patients with severe intermaxillary discrepancies}

\author{
LUCIANA MUNHOZ \\ Prof. Dr. Roberto Heitzmann Rodrigues Pinto 2 \\ Profa Dra Emiko Saito Arita ${ }^{3}$
}

\begin{abstract}
RESUMO
Objetivo: Buscou-se neste artigo apresentar um posicionador radiográfico para radiografias periapicais intraorais de dentes anteriores modificado que viabiliza a estabilização do posicionador pela oclusão de pacientes com discrepâncias intermaxilares severas.

Métodos: Foi construído um protótipo que apresenta área de oclusão rígida alongada. Dois pacientes portadores de retrognatismo severo, associado ou não à presença de inclinações dentárias marcantes, demonstraram o posicionador simulando o momento da exposição radiográfica.

Resultados: Observou-se que os pacientes conseguiram melhor estabilidade ao ocluir o posicionador proposto em detrimento aos disponíveis no mercado odontológico.

Conclusões: O posicionador desenvolvido apresentado pode ser eficiente na obtenção de imagens radiográficas periapicais intraorais para pacientes com discrepâncias intermaxilares severas, sejam elas de origem esquelética ou dentárias, pois possui área de oclusão rígida alongada em relação aos demais dispositivos disponíveis no mercado.
\end{abstract}

Palavras-chave: Posicionador radiográfico. Radiologia odontológica. Radiografia intraoral.

\begin{abstract}
Objective: To present a modified intraoral periapical film holder for anterior teeth that allows its stabilization by the occlusion of patients with severe intermaxillary discrepancies.

Methods: A prototype that presents an area of elongated rigid occlusion was developed. Two patients with severe retrognathism, associated or not to the presence of marked dental inclinations, demonstrated the film holder simulating the moment of the radiographic exposure.

Results: It was observed that the patients achieved better stability with the proposed film holder than with currently available film holders.

Conclusions: The film holder developed can be efficient in obtaining intraoral periapical radiographic images for patients with severe intermaxillary discrepancies, whether of skeletal or dental origin, because it has a rigid area of elongated to occlusion in relation to other devices available in the market.
\end{abstract}

Key-words: Radiographic film holder. Dental radiology. Intraoral radiography.

Mestranda em Diagnóstico Bucal, Departmento de Estomatologia, Faculdade de Odontologia da Universidade de São Paulo Professor do curso de especialização em Radiologia e Imaginologia Odontológica, Fundação para o Desenvolvimento Científico e Tecnológico da Odontologia (Fundecto)

3 Professora Associada, Departamento de Estomatologia, Faculdade de Odontologia da Universidade de São Paulo Av. Lineu Prestes, 2227. Cep: 05508-000. São Paulo, SP, Brasil. Telefone : +55 11 3091-7831. E-mail: dra.lucimunhoz@usp.br 


\section{INTRODUÇÃO}

As radiografias intraorais periapicais odontológicas são amplamente utilizadas por cirurgiões dentistas; constituem um método imagenológico de execução técnica simples, provedor de grande riqueza de detalhes do órgão dentário, periodonto e estruturas adjacentes. Ademais, tem importante função como documentação para o dentista e para o paciente, visto que o exame radiológico também é utilizado em odontologia legal. ${ }^{1}$ Contudo, para que sirvam ao seu propósito, devem apresentar mínima distorção. ${ }^{2}$ Entre as técnicas radiográficas intraorais periapicais, a técnica radiográfica do paralelismo é amplamente utilizada pelos cirurgiões dentistas. Baseia-se no princípio do paralelismo entre o longo eixo de implantação do dente e o longo eixo do filme radiográfico, sendo o último fixado por um suporte portafilme denominado posicionador radiográfico. $\mathrm{O}$ uso de tais posicionadores permite que o filme radiográfico mantenha-se localizado mais posteriormente à face lingual ou palatal dos dentes e o feixe de raios $X$ seja direcionado perpendicularmente ao filme, resultando na produção de imagens radiográficas com mínimas distorções geométricas. ${ }^{3}$ Os raios $X$ devem sempre atingir o filme nos ângulos corretos, de acordo com as teorias de projeção geométrica. Um pré-requisito para a exposição intraoral odontológica é a utilização de um posicionador que garanta que o feixe central de raios $X$ permaneça em ângulos corretos um em relação ao outro. O objeto e o filme devem estar o mais paralelo possível entre si. . $^{4,5}$

A utilização dos posicionadores radiográficos data do século $X X$ e está presente desde o início do uso dos raios $\mathrm{X}$ para diagnóstico em odontologia. Eles surgiram com a necessidade de estandardização das imagens radiográficas. . $^{6,7}$ Inicialmente, utilizavam os dedos do paciente ou do próprio profissional para segurar o filme radiográfico na cavidade oral do paciente, ${ }^{8}$ o que não permitia tal padronização. Outros aparatos, com a finalidade de melhorar a qualidade e minimizar as distorções produzidas na imagem da película radiográfica, como rolos de algodão, blocos de mordida e pinças hemostáticas também já foram sugeridos. ${ }^{9}$

Há uma enorme gama de posicionadores vendidos no mercado; eles possuem vantagens e desvantagens um em relação ao outro. ${ }^{1}$ A escolha dos dispositivos é uma questão de preferência pessoal e do tipo de receptor de imagem utilizado (filme ou sensor). ${ }^{10}$ Basicamente, esses são constituídos de três partes: mecanismo para manutenção do receptor de imagem paralelo ao dente não permitindo a sua curvatura; bloco ou plataforma de mordida (ou como designado nesse modelo demonstrativo, áreas de oclusão), e anel localizador (ou aro). ${ }^{10,11,12,13}$

A utilização de posicionadores radiográficos influencia diretamente na qualidade técnica das radiografias periapicais, quando permite que o cirurgião dentista posicione adequadamente o filme em relação ao longo eixo do dente sem preocupação em observar rigidamente os ângulos de incidência dos feixes de raios $X$ (pois basta posicionar o cilindro seguindo a orientação do aro do posicionador) ou se o paciente conseguirá segurar o filme conforme instruções do profissional. Outras vantagens da utilização de posicionadores radiográficos estão relacionadas à possibilidade de obtenção de mensurações dentárias próximas ao tamanho real, ${ }^{14}$ prevenção do encurvamento do filme pela pressão dos dedos do paciente durante a exposição e a não necessidade de manutenção de posturas rígidas da cabeça do paciente seguindo planos anatômicos, facilitando o trabalho do profissional durante a execução da técnica. ${ }^{3}$ Cabe ressaltar que outros fatores também são tão relevantes na qualidade técnica dessas radiografias quanto 
POSICIONADOR RADIOGRÁFICO PERIAPICAL PARA PACIENTES COM DISCREPÂNCIAS INTERMAXILARES SEVERAS.

à aquisição das imagens radiográficas, como o processamento e o armazenamento das películas radiográficas. . $^{3,10,14}$

As dificuldades nas exposições radiográficas são inerentes às técnicas, exigindo do cirurgião dentista um domínio adequado dos procedimentos para a sua correta execução. ${ }^{15}$ As maiores preocupações do profissional durante a execução de radiografias intraorais são evitar a exposição excessiva do paciente às radiações ionizantes e a distorção da imagem dos órgãos dentários e estruturas adjacentes. ${ }^{16}$ Os cirurgiões dentistas devem objetivar a utilização de todos os meios necessários para diminuir a exposição à radiação pelo paciente, evitando repetições por meio do aperfeiçoamento das técnicas clínicas sem prejuízo à qualidade do diagnóstico. ${ }^{16}$ Posicionadores radiográficos reduzem a indução ao erro, evitando exposições desnecessárias do paciente, como preconizado pela portaria 453 do Ministério da Saúde ${ }^{17}$ e de acordo com os princípios de ALARA (As Low As Reasonably Achievable). ${ }^{18}$ A American Dental Association também recomenda o emprego de posicionadores, evidenciando sua importância ao alinhar o filme radiográfico com o cone colimador, evitando repetições. ${ }^{19}$ Existe a necessidade de se conhecer a correta utilização de cada dispositivo. ${ }^{14}$

Todavia, mesmo com a utilização de tais dispositivos, pode-se não obter radiografias satisfatórias, pela dificuldade de manuseio na execução da técnica ou falta de colaboração do paciente. ${ }^{1,20}$ Erros comumente observados na clínica radiológica incluem a colocação incorreta do filme no posicionador, exposição incompleta do filme (formação de "meialua"), alterações nas angulações, falta de centralização da região/dente a ser radiografado (a), ${ }^{21}$ além de fatores inerentes ao próprio paciente como movimentação durante a exposição (por dificuldade de estabilização do posicionador ou outros motivos). Um posicionador radiográfico que preencha todos os requisitos para uma radiografia perfeita ainda não foi criado. ${ }^{1}$

Os dispositivos são estabilizados pela oclusão do paciente. Nos dentes anteriores, as faces incisais dos dentes a serem radiografados ocluem em uma área semirrígida semirrígida, confeccionada em silicone ou etil vinil acetato, e as bordas incisais dos dentes antagonistas devem ocluir em uma área rígida e denticulada. Em pacientes com discrepâncias severas entre os arcos superior e inferior, tal estabilização pode ser dificultada. Pacientes com prognatismo ou retrognatismo severos, assim como inclinações acentuadas de dentes anteriores superiores em relação aos inferiores, podem apresentar dificuldades para estabilizar os posicionadores radiográficos convencionais; daí a necessidade de introdução de modificações nestes visando possibilitar a correta estabilização dos posicionadores para a obtenção de uma radiografia periapical correta e devidamente padronizada.

\section{Proposição}

O objetivo deste artigo foi apresentar um posicionador radiográfico para dentes anteriores superiores e inferiores modificado que atenda às necessidades de pacientes portadores de discrepâncias intermaxilares severas, comparando-o com os atuais modelos disponíveis no mercado, simulando um momento de exposição radiográfica em pacientes portadores de retrognatismo severo para os quais o uso dos dispositivos disponíveis no mercado apresentou-se insatisfatório.

\section{Materiais e mÉtodos}

O posicionador radiográfico modificado para pacientes com discrepâncias intermaxilares é composto, conforme observado na Figura 
1, por partes denominadas aro, haste, área de oclusão rígida com encaixe para bordas incisais, prendedor de filme radiográfico intraoral e área de oclusão rígida oposta. Note-se que ambas as áreas de oclusão apresentam serrilhas, paralelas entre si, que visam o encaixe das bordas incisais dos dentes anteriores, garantindo maior estabilização do mesmo. Os materiais selecionados para a confecção do posicionador não interferem na imagem radiográfica obtida, após a exposição do filme e as estruturas biológicas aos raios $X, e$, adicionalmente, são passíveis de desinfecção e/ou esterilização. Ademais, o prendedor de filme radiográfico é mais estreito, propiciando maior conforto ao paciente.

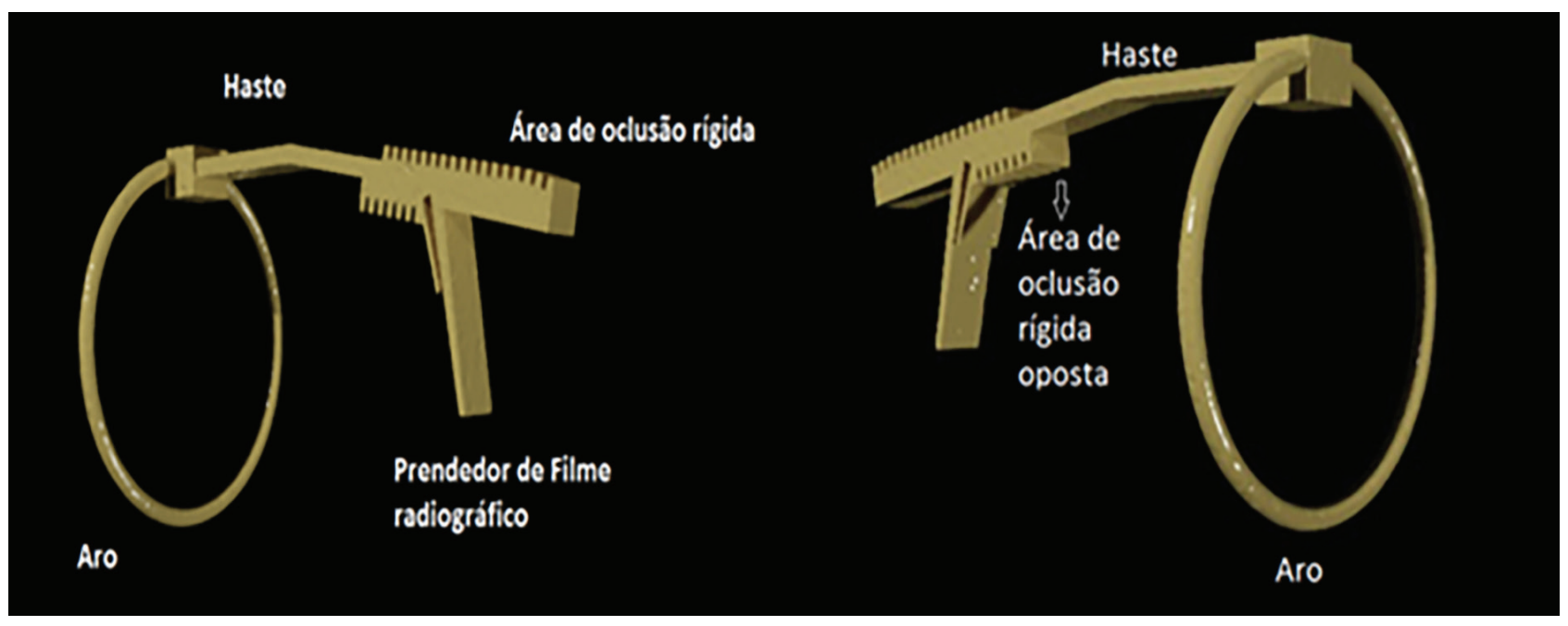

Figura 1: Desenho esquemático do posicionador proposto, com descrição das partes que o compõem.

Para demonstrar a utilização desse posicionador, foram utilizados dois pacientes modelos, portadores de retrognatismo severo, associado ou não à presença de inclinações dentárias marcantes, como visto na Figura 2. Foram feitas fotografias dos posicionadores em oclusão simulando o momento da exposição radiográfica. Foi analisada a estabilidade do posicionador proposto, obtida por meio da oclusão do paciente, comparando-o com outros disponíveis no mercado da marca Indusbello (Londrina, Paraná, Brasil) e Jon (São Paulo, São Paulo, Brasil), apresentados na Figura 3. Analisou-se especialmente as diferenças nos comprimentos das áreas de oclusão rígida e a possibilidade de oclusão por parte do paciente.

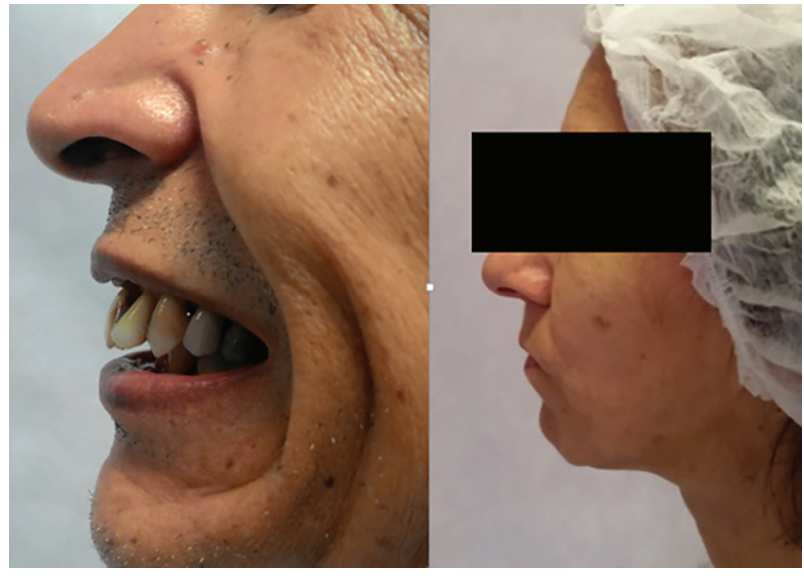

Figura 2: Pacientes modelos. Da esquerda para a direita, o primeiro paciente, classe 2, apresenta vestibularização marcante dos incisivos superiores. O segundo paciente, também classe 2 , contraindo a musculatura do mento para obter vedamento labial. 
Nota: o posicionador também pode ser utilizado em pacientes portadores de má oclusão classe 3, ou mesmo com inclinações dentárias excessivas que resultem em descompensação entre os arcos.

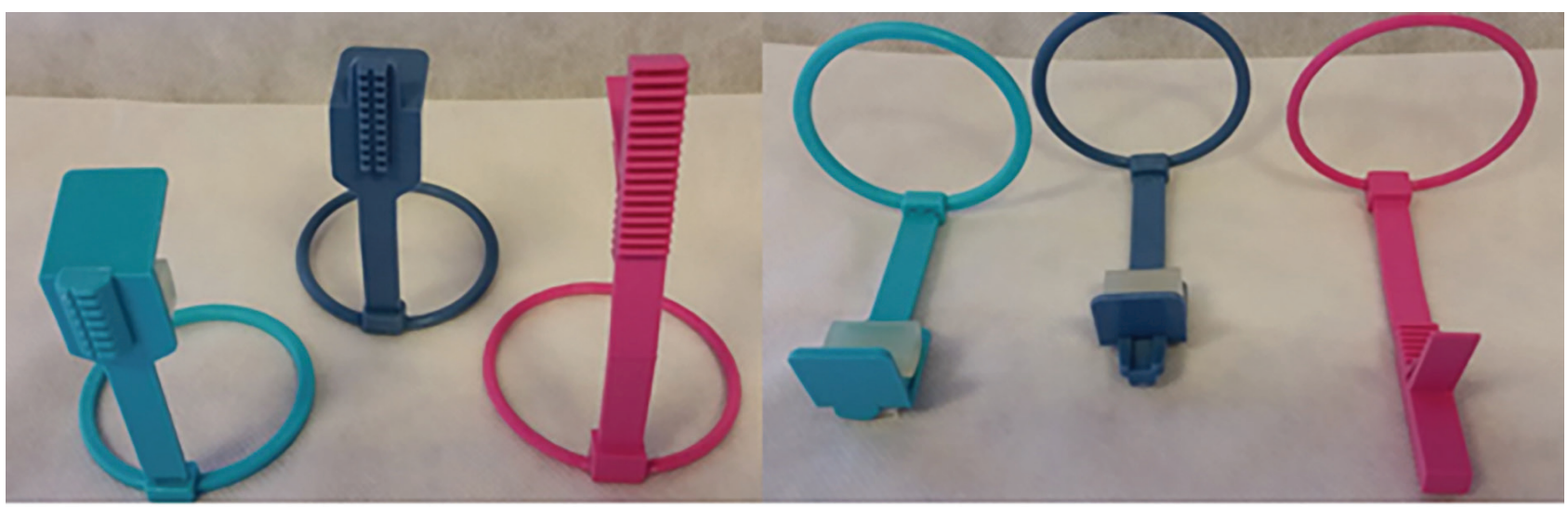

Figura 3: Posicionadores radiográficos presentes no mercado para uso em odontologia. Em verde, o posicionador da marca Jon, em azul da marca Indusbello e em cor-de-rosa o protótipo final idealizado.

\section{Resultados}

Observou-se que uma paciente modelo não conseguiu estabilizar pela oclusão os posicionadores radiográficos intraorais para dentes anteriores das marcas Jon ou Indusbello. Foi criada uma extensão em resina acrílica para o posicionador Indusbello para as fotos. Nota-se que os pacientes não são capazes de ocluírem na área representada originalmente pelo posicionador (em azul), ocorrendo o mesmo com o segundo paciente (Figura 4). A extensão em resina acrílica foi criada unicamente para o registro das fotos, visando evidenciar a necessidade de extensão da área de oclusão rígida.
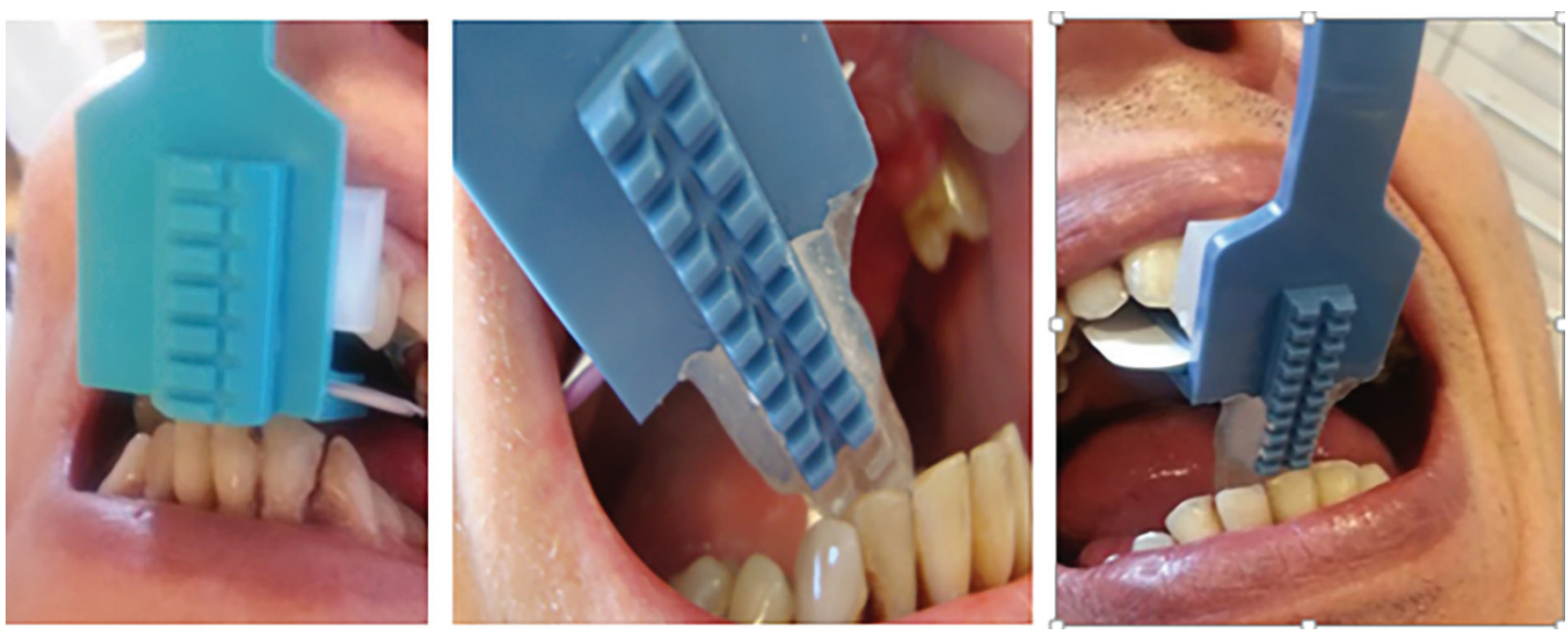

Figura 4: Da esquerda para a direita, na primeira imagem, nota-se impossibilidade de oclusão para o primeiro posicionador, com área de oclusão curta. Nas duas imagens da sequência, detalhe para a paciente ocluindo na extensão de resina acrílica; idem para a terceira imagem. 

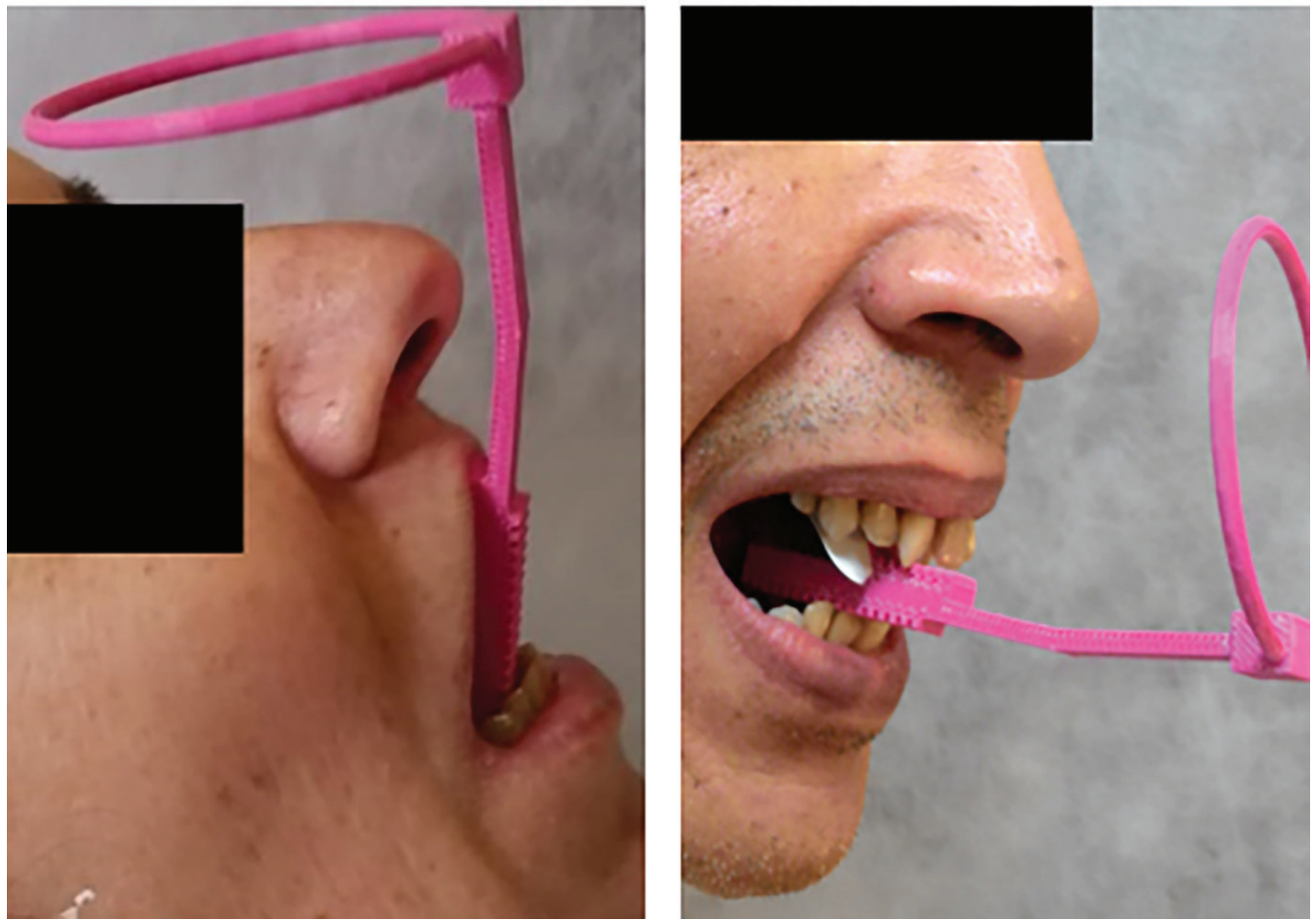

Figura 5: Protótipo do posicionador modificado simulando o momento da exposição radiográfica.

Os pacientes, todavia, conseguiram ocluir e estabilizar o dispositivo modificado no momento da simulação da exposição radiográfica, conforme demonstrado na Figura 5. A área de oclusão alongada em relação aos demais posicionadores foi a responsável pela estabilização do aparato modificado na cavidade oral do paciente.

\section{Discussão}

Posicionadores radiográficos intraorais periapicais são extremamente úteis e práticos na facilitação da obtenção de imagens radiográficas de boa qualidade, além de permitir a padronização das imagens obtidas. Sua utilização influencia diretamente na qualidade técnica das radiografias periapicais, eles possibilitam mensurações dentárias próximas ao tamanho real, ${ }^{14}$ previnem o encurvamento do filme pela pressão dos dedos do paciente, não exigem posturas rígidas de cabeça do paciente ${ }^{3}$ e evitam a exposição desnecessária dos dedos do paciente às radiações ionizantes durante a execução da técnica. Ademais, são preconizados pela portaria 453 do Ministério da Saúde ${ }^{17}$ e pelos princípios "As Low As Reasonably Achievable" $(\mathrm{ALARA})^{7}$ pois reduzem a indução ao erro e, consequentemente, repetições.

Apesar de todas as vantagens na utilização dos posicionadores e da variedade disponível no mercado, os mesmos possuem limitações. Esses são estabilizados pela oclusão do paciente no momento da exposição radiográfica. Particularmente sobre os posicionadores periapicais intraorais para dentes anteriores existentes no mercado, as bordas incisais dos dentes a serem radiografados tocam em uma área semirrígida, confeccionada em silicone atóxico, e as bordas incisais dos dentes antagonistas devem ocluir, mantendo o conjunto em posição em uma área rígida denticulada (designada, de 
acordo com a Figura 1, como área de oclusão rígida). Nesse modelo, a área semirígida foi substituída por uma área rígida, denticulada, para prover maior estabilidade (denominada, no dispositivo desenvolvido, como área de oclusão rígida oposta, de acordo com a Figura 2). A área de oclusão rígida da marca comercial JON e Indusbello (considerando-se apenas a área denticulada) possui dimensões menores do que a do posicionador proposto, sendo a da marca comercial JON a menor de todas (Figura 3). Embora suficientes para atender às necessidades de pacientes com relações intermaxilares harmônicas ou com discrepâncias leves à moderadas, o emprego dos mesmos em pacientes com discrepâncias intermaxilares severas (de origem esquelética ou dentária) é insuficiente. Assim, tornou-se essencial a construção de um posicionador específico para tais pacientes, propiciando a manutenção do conjunto posicionador e filme radiográfico imóvel durante a exposição, por meio da extensão da área de oclusão rígida.

O posicionador demonstrado neste artigo tem o diferencial de possuir uma área denticulada maior, alongada em relação aos demais, conforme comparativo na Figura 3. Cabe ressaltar que foram considerados como pacientes portadores de discrepância intermaxilar severa os pacientes portadores de inclinações dentárias excessivas (alterando a relação entre os arcos), prognatismo ou retrognatismo acentuados e aqueles que não conseguem ocluir na área denticulada dos dispositivos disponíveis no mercado pela ausência de dentes associada. O uso dos posicionadores padronizados disponíveis no mercado é aceitável contanto que o cirurgião dentista realize adaptações corretas e suficientemente rígidas, visando estender a área de oclusão rígida e, consequentemente, estabilizando a oclusão do paciente no momento da exposição radiográfica sem o auxílio de outros aparatos (como mãos, pinças mosquito etc.). Todavia, dependendo do material utilizado na adaptação, o profissional pode danificar seu dispositivo original, impossibilitando o uso do mesmo em outros pacientes sem discrepâncias intermaxilares e impedindo a esterilização posterior do posicionador.

Entretanto, mesmo com o uso de posicionadores devidamente imobilizados pela oclusão do paciente, a aquisição de imagens radiográficas satisfatórias depende de outros fatores primordiais como a colaboração do paciente e a execução acertada da técnica. ${ }^{1,20}$ Como já mencionado, um posicionador radiográfico que preencha todos os requisitos para uma radiografia perfeita ainda não foi criado, ${ }^{1}$ entretanto, melhorias podem ser introduzidas aos já existentes visando aprimorar a técnica radiográfica, beneficiando tanto os cirurgiões dentistas como os próprios pacientes.

\section{Considerações finais}

O posicionador desenvolvido apresentado pode ser eficiente na obtenção de imagens radiográficas periapicais intraorais para pacientes com discrepâncias intermaxilares severas, sejam elas de origem esquelética ou dentárias, pois possui área de oclusão rígida alongada em relação aos demais dispositivos disponíveis no mercado. Cirurgiões dentistas podem realizar adaptações em posicionadores já disponíveis no mercado, entretanto, pela necessidade de suporte rígido para manter a oclusão do paciente, tais adaptações podem alterar o posicionador permanentemente, impossibilitando o uso do mesmo em pacientes sem discrepância intermaxilar e impedindo a esterilização adequada posterior do aparato.

\section{Agradecimentos}

Aos senhores Antonio Munhoz Filho e Hermes Francisco dos Reis Neto pelos desenhos em Autocad 2 e 3D do protótipo apresentado. 


\section{REFERÊNCIAS}

1. Hayashi MM, Issa JPM, Tiossi R., Pardini LC, Watanabe PCA. Evaluation of the use of film holders by undergraduation students. Int $\mathbf{J}$ Odontostomat. 2007; 1(2): 109-114.

2. Coelho RM, Sumas KSR, Oliveira Júnior IS, Manzi FR. Comparação de posicionadores radiográficos considerando aspectos periodontais. Rev Period. 2007; 17(3): 80-85.

3. Fenyo-Pereira M. Radiologia Odontológica e Imaginologia, 2. ed. Ed Santos. São Paulo, 2012.

4. Fuhrmann AW. Current pratice in convencional and digital intraoral radiography: problems and solutions. Int J Comp Dent. 2006; 9: 61-88.

5. van Aken L., Verhoeven JW. Factors influencing the design of aiming devices for intraoral radiography and their practical application. Oral Surg Oral Med Oral Pathol. 1979; 47(4): 378388.

6. Dixon DA, Hildebolt CF. An overview of radiographic film holders. Dentomax Rad. 2005; 34: 67-73.

7. Dixon DA, Hildebolt CF. An overview of radiographic film holders. Dento maxillofacial Radiol. 2005; 34(2): 67-73.

8. Pitts NB. Film-holding, beam-aiming and collimating devices as an aid to standardization in intraoral radiography: a review. J Dent. 1984; 12: 36-46.

9. Hilu R., Arfuch A., Vietto L. Determinación de la longitud de trabajo em conductos radiculares, con la utilización del posicionador radiográfico Endo Ray II. RAOA. 2004; 92(2): 109-113.

10. Whaites E. Princípios de Radiologia Odontológica. Rio de Janeiro. Ed Elsevier, 2009, 4. ed.

11. Price CA. Adevice enabling the constant repetition of periapical radiographs. Dentomaxillofac Radiol. 1972; 1:15.

12. Wege WR. A technique for sequentially reproducing intraoral film. Oral Surg Oral Med Oral Pathol Oral Radiol Endod. 1967, 23: 454458.

13.Duinkerke AS, Poel AC, Van der Linden FP, Lemmens W. Evaluation of a technique for standardized periapical radiographs. Oral Surg
Oral Med Oral Pathol Oral Radiol Endod. 1977, 4: 646-651.

14. Terr ER, Pontual AA, Cruz AD, Haiter Neto F. Avaliação da precisão da imagem radiográfica obtida com o uso de diferentes posicionadores. Rev. Assoc. Paul. Cir. Dent. 2006; 60(5): 357360.

15.Schimitz MS, Montagner F., Ferreira G., Gomes BPFA. A utilização de posicionadores radiográficos na etapa trans operatória em endodontia: proposição de técnica. Rev Assoc Paul Cir Dent. 2010; 64(2): 139-143.

16. Choksi SK, Rao MS. Comparison of two film holders for periapical radiography performed by dental students. Maryland Dental State Assoc. 1996; 39(1): 23-39.

17. Brasil. Ministério da Saúde. Secretaria de Vigilância Sanitária. Portaria 453, de 1 de junho de 1998. Aprova o Regulamento Técnico que estabelece as diretrizes básicas da proteção radiológica em radiodiagnóstico médico e odontológico, dispõe sobre o uso dos raios $\mathrm{X}$ diagnósticos em todo território nacional e dá outras providências. Diário Oficial da União, Poder Executivo. Brasília (DF); 1998, jun. 2.

18. Bevelacqua JJ. Practical and effective ALARA. Health physics. 2010; 98 (Suppl 2): S39-47.

19. American Dental Association. Council on Scientific Affairs. The use of dental radiographs - an update and recommendations. J Am Dent Assoc. 2006; 137(9): 1.304-1.312.

20. Caldeira EJ, Camilli JA, Cagnon VH. Posicionador experimental para auxiliar radiografias periapicais. Rev Gaúcha Odontol. 2004; 52(2): 124-126.

21. Wu JC1, Huang JN, Zhao SF, Xu XJ, Zhang JC, Xia B, Dong YF. Use ofa simple intraoral instrument to standardize film alignment and improve imagereproducibility. Oral Surg Oral Med Oral Pathol Oral Radiol Endod. 2005, jul.; 100(1): 99-104.

Submetido em: 9-5-2017

Aceito em: 22-6-2017 